\section{Immunohistochemical expression of types I and III collagen antibodies in the temporomandibular joint disc of human foetuses}

\author{
L.O.C. de Moraes, ${ }^{1}$ F.R. Lodi, ${ }^{1}$ T.S. Gomes, ${ }^{2}$ \\ S.R. Marques, ${ }^{1}$ C.T.F. Oshima, ${ }^{2}$ \\ C.L.P. Lancellotti, ${ }^{3}$ \\ J.F. Rodríguez-Vázquez, ${ }^{4}$ \\ J.R. Mérida-Velasco, ${ }^{4}$ L.G. Alonso ${ }^{1}$ \\ 'Departamento de Morfologia e Genética, \\ Universidade Federal de São Paulo, \\ Brasil; \\ ${ }^{2}$ Laboratório de Patologia Molecular, \\ Departamento de Patologia, Universidade \\ Federal de São Paulo, Brasil; \\ ${ }^{3}$ Departamento de Ciências Patológicas, \\ Santa Casa de São Paulo, Brasil; \\ ${ }^{4}$ Departamento de Anatomía y \\ Embriología II, Universidad Complutense \\ de Madrid, España
}

\section{Abstract}

The objective was to study the morphology of the articular disc and analyse the immunohistochemical expression of types I and III collagen markers in the temporomandibular joint (TMJ) disc of human foetuses of different gestational ages. Twenty TMJ from human foetuses supplied by Universidade Federal de Uberaba with gestational ages from 17 to 24 weeks were studied. The gestational age of the foetuses was determined by measuring the crown-rump (CR) length. Macroscopically, the foetuses were fixed in $10 \%$ formalin solution and dissected by removing the skin and subcutaneous tissue and exposing the deep structures. Immunohistochemical markers of type I and III were used to characterize the existence of collagen fibres. Analysis of the immunohistochemical markers of types I and III collagen revealed the presence of heterotypical fibril networks.

\section{Introduction}

The temporomandibular joint (TMJ) is a joint of the sinovial, double and bicondilar type. It is situated between the condylar process and the mandibular fossa of the temporal bone. It has an articular capsule, synovial membrane, ligaments and associated muscles. ${ }^{1-4}$

In comparison with other synovial joints, the
TMJ is unique because of the presence of the articular disc that separates it into two independent cavities, known as infra and supradiscal cavities, with distinct morphological features. ${ }^{3,5}$ At the end of the $12^{\text {th }}$ gestational week, both the articular disc and the cavities are already totally organized. ${ }^{6,7}$

Morphologically, the articular disc is a small elliptic fibrocartilaginous plate shaped like an $\mathrm{S}$ in sagittal cut and having its bigger axis in the posteromedial direction. The articular disc is divided into four bands: anterior, intermediate, posterior and bilaminar zone. ${ }^{3,8}$

The disc also plays an important functional role in translation and rotation movements, in a broad and passive manner. The articular disc can be described as a concave lens with two faces (superior and inferior), two margins (medial and lateral) and two extremities (anterior e posterior). ${ }^{9,10}$

During the embryogenesis period, the articular disc of the TMJ consists of a dense layer of mesenchymal cells that derived from the condylar blastema in the anterior region and from the glenoidal blastema in the posterior region. ${ }^{6,7}$

For Sabú et al., the architecture of the articular disc is complex, with collagen fibres predominating in the longitudinal direction of the superior and inferior faces and cross-sectional and interposed oblique fibres predominating in the intermediate portion. ${ }^{11}$ For Minarelli and Liberti, the disc has compact collagen bands in the intermediate band, with fibres running in the anteroposterior direction. ${ }^{12}$

Takahashi and Sato examined the distribution of types I and III collagen, fibronectin and elastic fibres in the articular disc of 54 adult cadavers and 24 foetuses aged 12, 16, 20, 24, 28 and 32 weeks with light microscopy and scanning electron microscopy. According to the authors, at 12 weeks, the collagen fibres formed a thin layer on the entire disc; at 16 weeks, the disc already had anteroposteriorlyoriented collagen fibres in the intermediate band and cross-sectional fibres were found in the most superficial layers of the disc; finally, at 20 weeks, the authors found a disc consisting of a complex arrangement of collagen fibres, oriented in various directions. ${ }^{13}$

In terms of structural biology, the collagen fibres inside the articular disc of the TMJ are composed mainly of type I collagen and small amounts of type III collagen. ${ }^{3}$

According to Takahashi and Sato, the concentration of type-I collagens in the articular discs of foetuses aged 20 to 24 weeks increased in all bands, especially in the anterior and intermediate bands. Meanwhile, the expression of type-three collagen in foetuses of the same gestational age increased only in the intermediate band. ${ }^{13}$

Kiga et al. confirmed that a large number of
Correspondence: Luís Otávio Carvalho de Moraes, Departamento de Morfologia e Genética, Universidade Federal de São Paulo, Rua Botucatu, 740 - Edifício Leitão da Cunha (térreo), Vila Clementino - São Paulo, SP, 04023 900 Brasil.

Tel. +55.11:55764261-25 - Fax +55.11.55717597.

E-mail: lgalonso.ops@terra.com.br

Key words: articular disc, temporomandibular joint, human foetuses, collagen, immunohistochemical, embryology.

Contributions: LOCM, CLPL, TSG, CTFO laboratory experiments performing; LOCM, JFR-V, JRM-V results analysis and interpretation; LOCM, FRL, SRM, LGA, research theme defining, methods designing and manuscript writing. All authors approved the manuscript final version.

Acknowledgements: this investigation was supported by the Coordenação de Aperfeiçoamento de Pessoal de Nível Superior (CAPES, Brazil).

Conflict of interest: the authors report no conflicts of interest.

Received for publication: 7 February 2011. Accepted for publication: 24 May 2011.

This work is licensed under a Creative Commons Attribution NonCommercial 3.0 License (CC BYNC 3.0).

(OCopyright L.O.C. de Moraes et al., 2011

Licensee PAGEPress, Italy

European Journal of Histochemistry 2011; 55:e24 doi:10.4081/ejh.2011.e24

fibroblast-like cells in the human TMJ disc was co-localized with lumican expression in their specimens. ${ }^{14}$ In contrast, little lumican expression was observed in the vicinity of fibrochondrocytes and chondrocyte-like cells. Matsumoto et al. demonstrated the presence of HAS3 in the chondrocyte-like cells of both normal and deformed TMJ discs in adults. ${ }^{15}$

A study done at the Institute of Anatomy, University of Catania, Italy, used four articular discs from adult cadavers as controls. A multidirectional preservation of the collagen bands was found in all samples, in addition to a predominance of fibrocyte-like cells, and in a smaller aggregated amount, chondrocyte-like cells. ${ }^{16}$

The apparent presence of fibrocartilaginous areas also suggests the presence of type II collagen fibers. ${ }^{3}$

Fibrous proteins (collagen and elastin), responsible for the shape and structure of the lamina propria, are associated with resistance and elasticity, while interstitial proteins (glycoproteins and proteoglycans) control viscosity, hydration, and the tissue volume extracellular matrix..$^{15-18}$

According to Gray et al., the characteristics 
of the layers of the lamina propria are intimately associated with the arrangement and distribution of the fibrous proteins in the extracellular matrix, especially collagen fibres, which support and absorb the physical stress caused on the articular disc by joint movement. ${ }^{19}$

Collagen belongs to a family of structural proteins with distinct chemical compositions, morphologies and functions. Collagen is a component of many organs and tissues and is the main extracellular matrix element of multicellular animals, representing from 25-30\% of the protein mass of the organism..$^{20}$

More than 27 types of collagen have been classified. The different types of collagens have been organized into the following classes: fibrillar, associated with fibrils, non-fibrillar, filamentous, short- and long-chain. ${ }^{21}$

It has also been demonstrated that some types of collagen are capable of aggregating molecules to form heterotypical fibrils. The presence of heterotypical fibrils with different combinations in many tissues has already been reported. ${ }^{22,23}$ Evidence of these combinations obtained with immunohistochemical analysis has been observed between types I and $\mathrm{V}$ collagen in human skin, tendon and cornea $^{22,24}$ and between types II and XI, and between I and II in cartilage. ${ }^{25}$

Given the scarcity of studies that focus morphologically on the types of collagen in the TMJ disc of human foetuses, we believe a better structural knowledge of the relationship of these proteins with the articular disc of the TMJ is necessary. Thus, the objective of this study is to analyse the immunohistochemical expression of types I and III collagen markers in the articular disc of the temporomandibular joint of human foetuses.

\section{Materials and Methods}

The protocol of this study was submitted to the UNIFESP Research Ethics Committee and approved under number 0225/05.

Our sample consisted of 10 human foetuses supplied by the Universidade Federal de Uberaba, resulting in $20 \mathrm{TMJ}$ with gestational ages from 17 to 24 weeks. The gestational age of the foetuses was determined by measuring the crown-rump (CR) length. ${ }^{26}$

All foetuses were preserved in a $10 \%$ formalin solution and dissected by removing the skin, subcutaneous tissue and exposing the deep structures.

Two sections of $3 \mu \mathrm{m}$ were taken from each paraffin block containing the collected material and were then spread on glass slides. The streptavidin-biotin technique was used to analyse the immunohistochemistry reactions of types I and III collagens. Sections were deparaffinised in three changes of xylene (10 min each) and rehydrated in a graded series (100, 80 and 70\%) of ethanol finishing in distilled water. Antigen sites were retrieved by pretreatment with $0.5 \mathrm{M}$ acetic acid for $90 \mathrm{~min}$ at $37^{\circ} \mathrm{C}$ and multiple rinses with distilled water.

Endogenous peroxidases were quenched by incubating in $3 \% \mathrm{H}_{2} \mathrm{O}_{2}$ (20 min, room temperature). The slides were then washed $3 \times 2 \mathrm{~min}$ in PBS and incubated in milk powder solution for 20 min to block nonspecific binding of antibody. Sections were incubated with $100 \mu \mathrm{L}$ of the primary antibody overnight at $4^{\circ} \mathrm{C}$.Primary antibody was purified rabbit anti-human type I collagen, 1:800 (Novatec Inc., Baltimore, MD, USA) and rabbit monoclonal anti-human type III collagen (Novatec Inc.). Subsequently, slides were washed in PBS $(3 \times 2 \mathrm{~min})$, and
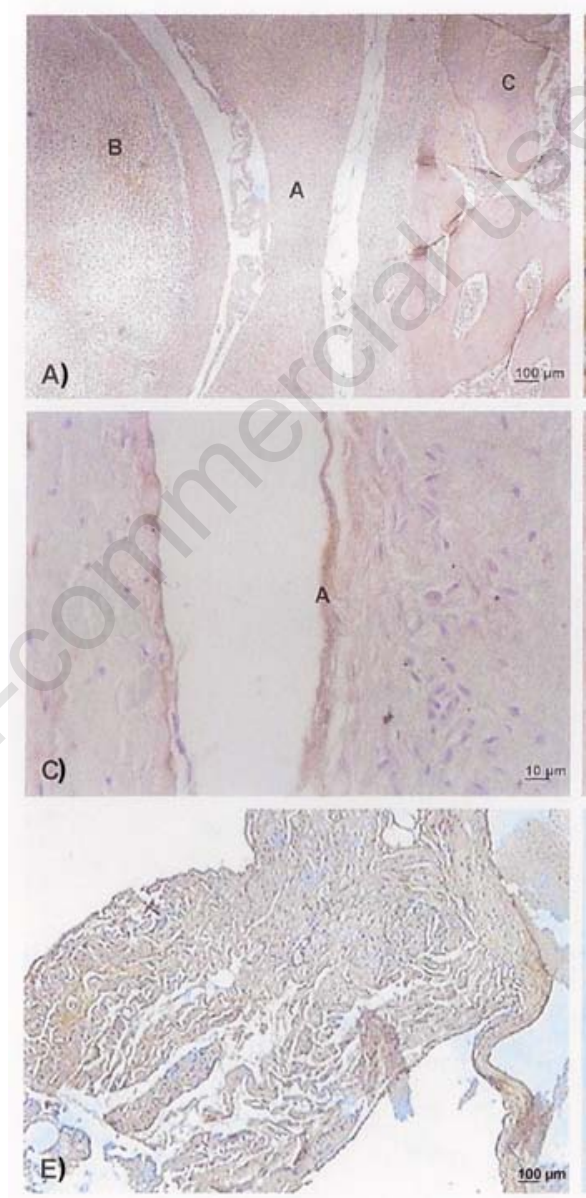

D)
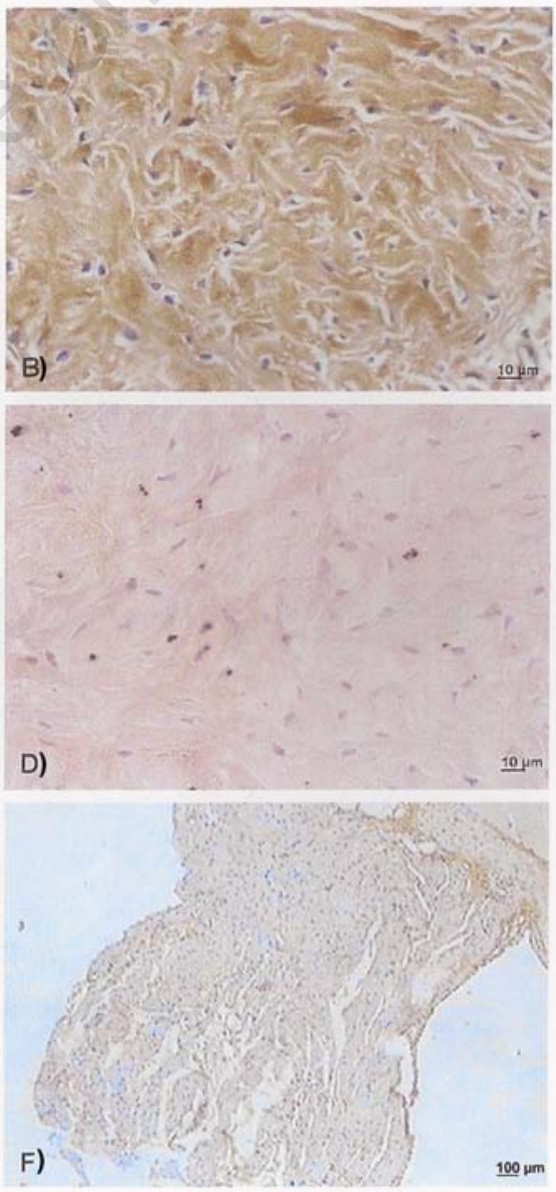

Figure 1. Immunohistochemical micrograph of type I collagen. A), foetus aged 19 weeks of intrauterine life; there are collagen fibres in the entire disc (A), mandibular head (B) and temporal bone (C); B), immunohistochemical photomicrograph of type I collagen in foetus at 22 weeks of intrauterine life; the posterior band of the articular disc contains numerous complexly-arranged collagen fibres; C), immunohistochemical micrograph of type III collagen in foetus at 21 weeks of intrauterine life; The inferior face (A) of the articular disc is delimited by numerous collagen fibres; $\mathrm{D}$ ), immunohistochemical micrograph of type III collagen in foetus at $\mathbf{2 4}$ weeks of intrauterine life; collagen fibres are arranged in many directions on the inferior face (intermediate). The heart tissues used as positive controls for collagen I and III are represented in panels E) and F), respectively. 
The negative controls were the same cases used as positive controls, which were submitted to the immunohistochemical reaction described above, except for the incubation with the primary antibody, done with a buffer solution.

\section{Results}

Figure 1A shows numerous type I collagen fibres throughout the extension of the articular disc of the TMJ. However, these fibres are more abundant in the posterior band of articular disc (1B). Additionally, Figure 1C shows a large concentration of type III collagen fibres on the inferior face of the articular disc. Figure 1D shows type III collagen fibres in the intermediate band of the articular disc. The positive controls are shown in Figures 1E and 1F.

The gestational ages of the foetuses are between 19 and 24 weeks.

\section{Discussion}

Minarelli and Liberti have a stated that the anterior band contains $85 \%$ of type I collagen fibres and $15 \%$ of type III collagen fibres, while the posterior contain $94 \%$ of type I collagen fibres and $6 \%$ of type III collagen fibres. ${ }^{12}$ Berkovitz et al. consider that the fibres inside the disc are composed mainly of type I collagen and are responsible for roughly $80 \%$ of the dry mass of the disc. Smaller amounts of type III collagen are also present. ${ }^{3}$

Figures $1 \mathrm{~A}$ and $1 \mathrm{~B}$ show numerous type I collagen fibres throughout the TMJ disc, especially in the posterior band. Figure $1 \mathrm{C}$ shows a great concentration of type III collagen fibres located on the inferior face of the disc, while Figure 1D shows complexly arranged type III collagen fibres running in many different directions. This does not mean that the anterior, intermediate and posterior bands of the disc were not marked by the antibody, but when type I collagen fibres are compared with those of type III, there is a prevalence of type I in the posterior band of the disc and a prevalence of type III on the entire inferior face of the disc.

In foetuses and children, type I collagen fibres have a stratified arrangement, organized in fibre bundles with an anteroposterior, laterolateral and oblique orientation forming mesh. ${ }^{11}$ In the posterior band of the disc, in agreement with our findings, the laterolateral fibre bundles were marked and, in agreement with Minarelli and Liberti, they formed a ring that surrounded the disc. ${ }^{12}$ In contrast, in the intermediate band of the disc, there was a prevalence of longitudinally oriented fibres in the anteroposterior direction. This orientation has been described by other authors in previous stdies. . $^{311,13,27}$

Descriptions of the arrangement that constitute the architecture of the articular disc have been made in humans of different ages and also in primates..$^{28,29}$

Again regarding the composition of collagen fibres, it is important to point out that the aggregation of fibrillar collagen molecules to form heterotypical fibrils has already been described in many structures such as skin, tendon, meniscus, cartilage, human cornea and heart. $3,22,23,25,30$

In our study we observed that these heterotypical fibrils had probably formed, in agreement with Takahashi and Sato ${ }^{13}$ who demonstrated that the disc of foetuses of various gestational ages contains types I and III collagens, and probably type II, as judged Berkovitz et al. ${ }^{3}$ by the presence of fibrocartilaginous areas in the articular disc.

The distribution pattern of the fibres throughout the foetuses' articular discs is different from that observed in adults' articular discs. $^{13}$ The following hypothesis may be considered: for the distribution patterns of types I and III collagens to change as the foetus develops and in adults, some events, such as sucking, small mandibular movements and load, begin during the maturation stage, after the twelfth week of development, and are lifelong.

Thus, findings from this study may lead us to conclude that immunohistochemical expression of types I and III antibodies is positive for the presence for these collagen fibres and heterotypical fibril meshes form between those two types of collagen.

\section{References}

1. Testut L, Latarjet A. Compendio de Anatomía Descriptiva, 22nd ed. 2006, Elsevier Masson, Barcelona, Spain.

2. Gardner E, Gray DJ, Rahilly R0. Anatomia: estudo bandal do corpo humano, 4th ed. 1988, Guanabara Koogan, Rio de Janeiro, Brazil.

3. Berkovitz BKB, Holland GR, Moxham BJ. Oral Anatomy, Histology and Embryology, 4th ed. 2009, Mosby, St. Louis, MD, USA.

4. Sabú C, Moraes LOC, Junior JAF, Oshima C, Smith RL, Alonso LG, et al. Ultra-structural evidence of vascularization of the central of TMJ disc in human fetuses. Eur J Anat 2006;10:45-8.

5. Mérida-Velasco Jr. New anatomical findings in the TMJ. An R Acad Nac Med 1999;115:981-7.

6. Van der Linden EJ, Burdi AR, Jongh HJ.
Critical periods in the prenatal morphogenesis of the human lateral pterygoid muscle, the mandibular condyle the articular disc and medical articular capsule. Am J Orthod Dentofac Orthop 1987;91:22-8.

7. Mérida-Velasco JR, Rodríguez-Vázquez JF, Mérida-Velasco JA, Sánchez-Montesinos I, Espín-Ferra J, Jiménez-Collado J. Development of the human temporomandibular joint. Anat. Rec. 1999;255:20-33.

8. Minarelli AM, Liberti EA. A microscopic survey of the human TMJ disc. J Oral Rehabilitation 1997;24:835-40.

9. Okeson JP. Fundamentos de oclusão e desordens temporomandibulares, 4th ed. 2000, ArtMed, São Paulo, Brazil.

10. Latarjet M, Ruiz-Liard A. Anatomía humana, 4th ed. 2007, Panamericana, Madrid, Spain.

11. Sabú C, Moraes LOC, Harb LJC, Junior JAF, Smith RL, Alonso LG, et al. Morphology and ultra-structure of the TMJ disc in human fetuses 21 to 28 weeks old. Braz J Morphol Sci 2005;22:129-36.

12. Minarelli AM, Liberti EA. The structure of the human temporomandibular joint disc: a scanning electron microscopy study. J Orofacial Pain 1997;11:95-100.

13. Takahashi H, Sato I. Ultrastructure of collagen fibers and distribution of extracellular matrix in the temporomandibular disk of the human fetus and adult. Okajimas Folia Anat Jpn 2001;78:211-22.

14. Kiga N, Tojyo I, Matsumoto T, Hiraishi Y, Shinohara Y, Fujita S. Expression of lumican related to CD34 and VEGF in the articular disc of the human temporomandibular joint. Eur J Histochem 2010;54:e34.

15. Matsumoto T, Inayama M, Tojyo I, Kiga N, Fujita S. Expression of hyaluronan synthase 3 in deformed human temporomandibular joint discs: in vivo and in vitro studies. Eur J Histochem 2010;54:e50.

16. Loreto C, Almdeida LE, Migliore MR, Caltabiano M, Leonardi R. TRAIL, DR5 and caspase 3-dependent apoptosis in vessels of diseased human temporomandibular joint disc. An immunohistochemical study. Eur J Histochem 2010;54:e40.

17. Gray SD, Hirano M, Sato K. Molecular and cellular structure of vocal fold tissue, 3rd ed. 1993, Singular, San Diego, CA, USA.

18. Pawlak AS, Hammond T, Hammond E, Gray SD. Immunocytochemical study of proteoglycans in vocal folds. Ann Otol Rhinol Laryngol 1996;105:6-11.

19. Gray SD, Titze IR, Chan R, Hammond TH. Vocal fold proteoglycans and their influence on biomechanics. Laryngoscope 1999;109:845-53.

20. Alberts B, Johnson A, Lewis J, Raff M, Roberts K, Walter P. Molecular Biology of the Cell, 5th ed. 2007, Garland Publ., 
London, UK.

21. Kadler KE, Canty EG, Lu Y. Matrix fully loaded - Assembly and secretion of collagen fibrils. Biochemist 2003;11-3.

22. Hendrix MJ, Hay ED, Von Der Mark K, Linsenmayer TF. Immonohistochemical localization of collagen types I and II developing chick cornea and tibia by electron microscopy. Ophthalmol Visc Sci 1982;22: 359-75.

23. Linsernamayer TF, Fitch JM, Birk DE. Heterotypic collagen fibrils and stabilizing collagens: controlling elements in corneal morphogenesis. Ann N Y Acad Sci 1990; 580:143-60.
24. Birk DE, Fitch JM, Babiarz JP, Doane KJ, Linsenmayer TF. Collagen fibrollogenesis in vitro interaction of types I and V collagen regulates fibril diameter. J Cell Sci 1990;95:649-57.

25. Chen Q, Fitch JM, Gibney E, Linsenmayer TF. Type II collagen during cartilage and corneal development: immunohistochemical analysis with an anti-telopeptide antibody. Dev Dyn 1993;196:47-53.

26. O'Rahilly R, Müller F. Human Embryology and Teratology, 3rd ed. 2001, Wiley-Liss, New York, NY, USA.

27. Ohta Y, Suwa F, Yang L, Wang M, Wang H. Development and histology of fibrous architecture of the fetal temporomandibular joint. Okajimas Folia Anat Jpn 1993;70:1-6.

28. Zerlotti E, Bernaba JM, Hetem S. Orientação das fibras colágenas do disco articular da articulação temporomandibular do sagüi. Rev Fac Odont Araçatuba 1972;1:91-5.

29. Taguchi N, Nakata S, Oka T. Three-dimensional observation of the temporomandibular joint disc in the rhesus monkey. J Oral Surg 1980;38:11-5.

30. McDevitt CA, Webber RJ. The ultra-structure and biochemistry of meniscal cartilage. Clin Orthop Relat Res 1990;252:8-18. 